\title{
Sección especial - Percepción de la biodiversidad
}

En la $25^{\circ}$ Reunión Argentina de Ecología (Luján, 2012) se llevó a cabo el Simposio "Percepción y conocimiento de la biodiversidad: ¿por qué y para qué incluir voces y miradas de múltiples actores sociales?". En este simposio gran parte de los grupos de trabajo de Argentina que estudian las relaciones entre las poblaciones humanas y la biodiversidad mostraron los avances de sus investigaciones e identificaron los vacíos de conocimiento que aún existen.

En agosto de 2013, en el marco del Congreso de la Asociación Internacional de Ecología (INTECOL), la profesora Georgina Mace, presidente dela Sociedad Británica de Ecología, disertó acerca de las tendencias históricas en la biología de la conservación. Remarcó que, en sus comienzos, la biología de la conservación se orientó hacia el estudio de la naturaleza en sí misma ("nature for itself") hasta que en la década del 80 empezó a considerarse la naturaleza "a pesar de" la gente ("nature despite people"). A comienzos del siglo XXI, con el estudio de las funciones y servicios ecosistémicos, la biología de la conservación se enfocó en el estudio de la naturaleza para la gente ("nature for people"). En estos últimos años, la tendencia está cambiando, y la conservación se centra en el estudio de las personas y la naturaleza ("people and nature"), considerando los sistemas socioecológicos.

En el escenario actual de pérdida continua de hábitats y biodiversidad, la conservación no puede limitarse solamente al establecimiento de áreas protegidas o a la creación de regulaciones legales para el uso de los recursos naturales. Se necesita contar con el apoyo de las comunidades humanas, considerando la diversidad de percepciones, apreciaciones, valoraciones y actitudes que ellas tienen hacia la biodiversidad. Por este motivo, surge a nivel mundial un llamado dirigido a los biólogos de la conservación instándolos a ser más proactivos en la tarea de intercambiar y compartir saberes mediante diferentes medios y con públicos diversos (Pace et al. 2010; Bickford et al. 2012).

En esta sección especial presentamos cinco trabajos que analizan las percepciones, valoraciones y conocimientos de diferentes actores sociales en relación con la biodiversidad. En el primer estudio, Mariela Borgnia y colaboradores abordan el problema de la introducción de especies exóticas y la importancia de incluir la diversidad de percepciones, opiniones y actitudes de distintos sectores de la sociedad a la hora de decidir e implementar las acciones de manejo y control, usando como caso de estudio la introducción de la ardilla de vientre rojo en Argentina. En las últimas décadas, ha aumentado el interés sobre los saberes etnobiológicos de los pueblos originarios ya que se considera que dichos conocimientos pueden contribuir a la conservación de la biodiversidad y el uso sustentable de los recursos naturales. En el segundo trabajo de esta sección, Ana Wawrzyk investiga los saberes de los pobladores de la puna jujeña sobre las vicuñas silvestres, que se basan en prácticas y vivencias vinculadas con el pastoreo y en las particularidades de la cosmovisión andina. En el tercer estudio, Adriana Rovere y colaboradores analizan cómo factores históricos y socioculturales afectan la preferencia de plantas que son utilizadas como cercos vivos en ciudades turísticas de la Patagonia. La influencia de la creación de los Parques Nacionales del sur del país y el poblamiento basado en una inmigración principalmente europea se manifiesta en el desarrollo cultural y paisajístico de la región. En el cuarto trabajo, Claudia Campos y colaboradores, revisn los estudios acerca de las percepciones, apreciaciones y conocimientos sobre la biodiversidad por parte de niños y adolescentes de zonas áridas de Argentina, considerando sus diferencias de género, edad y lugar de residencia. La poca familiaridad con las especies nativas por parte de estos jóvenes muestra la necesidad de concentrar esfuerzos en la educación formal y no formal relacionada con el conocimiento de la biodiversidad. Finalmente, Marina Richeri y coautores analizan el conocimiento ecológico tradicional y la flexibilidad ante los cambios ambientales de comunidades pequeñas de ascendencia criolla y mapuche-tehuelche de la patagonia. Los pobladores encuentran soluciones a los problemas locales aplicando sus saberes tradicionales junto con innovaciones prácticas en el uso de vegetales sustanciales para la vida cotidiana como las plantas leñateras y las medicinales. 
Agradecemos la invitación y el acompañamiento de Ecología Austral para la publicación de esta sección especial. El trabajo conjunto de autores, revisores y editores permitió la conformación de esta sección que esperamos sea de interés para todos aquellos que de una $u$ otra manera trabajan con las interacciones que ocurren en los sistemas socioecológicos.

Claudia M. Campos

(IADIZA - CONICET)

\section{REFERENCIAS}

Bickford, D; MRC Posa; L QIe; A Campos-Arceiz \& EP Kudavidanage. 2012. Science communication for biodiversity conservation. Biol. Conserv., 151: 74-76.

Pace, ML; SE Hampton; KE Limburg; EM Bennett; EM CoOK; AE Davis; JM Grove; KY Kaneshiro; SL LaDeau; GE LIKENS; DM MCKNIGHT; DC RICHARDSON \& DL STRAYER. 2010. Communicating with the public: opportunities and rewards for individual ecologists. Front. Ecol. Environ., 8: 292-298. 